Supplement of Hydrol. Earth Syst. Sci., 24, 2303-2322, 2020

https://doi.org/10.5194/hess-24-2303-2020-supplement

(C) Author(s) 2020. This work is distributed under

the Creative Commons Attribution 4.0 License.

(c) (1)

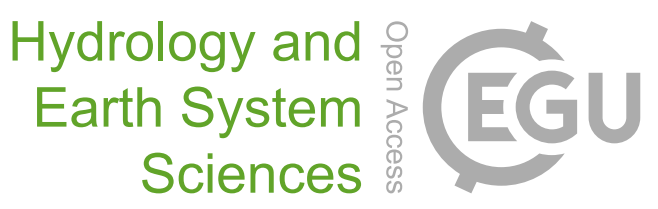

Supplement of

\title{
The pantropical response of soil moisture to El Niño
}

Kurt C. Solander et al.

Correspondence to: Kurt C. Solander (ksolander@lanl.gov)

The copyright of individual parts of the supplement might differ from the CC BY 4.0 License. 


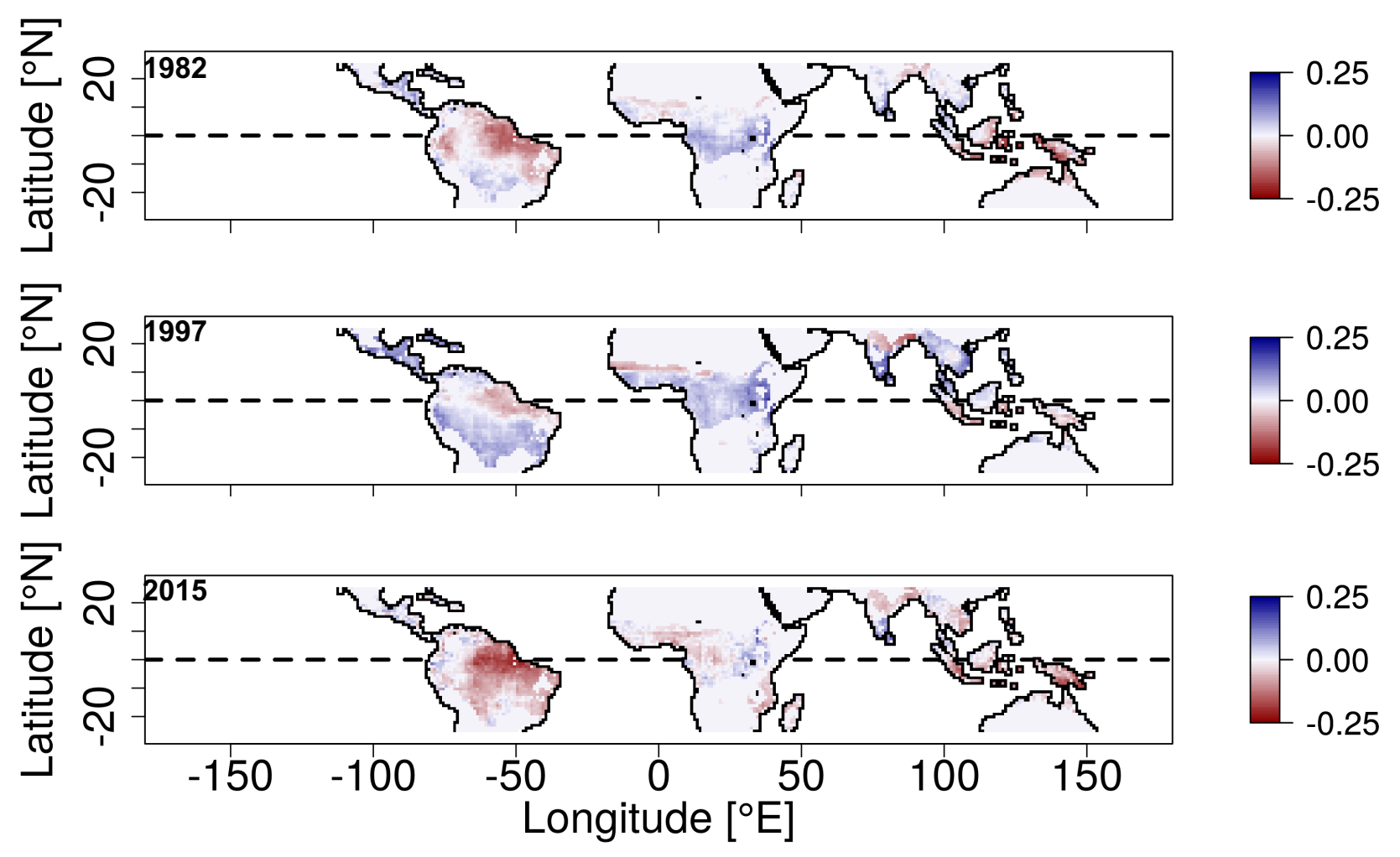

Figure S1a: October to December (OND) change in GLDAS soil moisture anomalies during the super El Niño years 1982 (top), 1997 (middle), and 2015 (bottom) relative to the previous years. Anomalies relative to 1979-2016 period. 


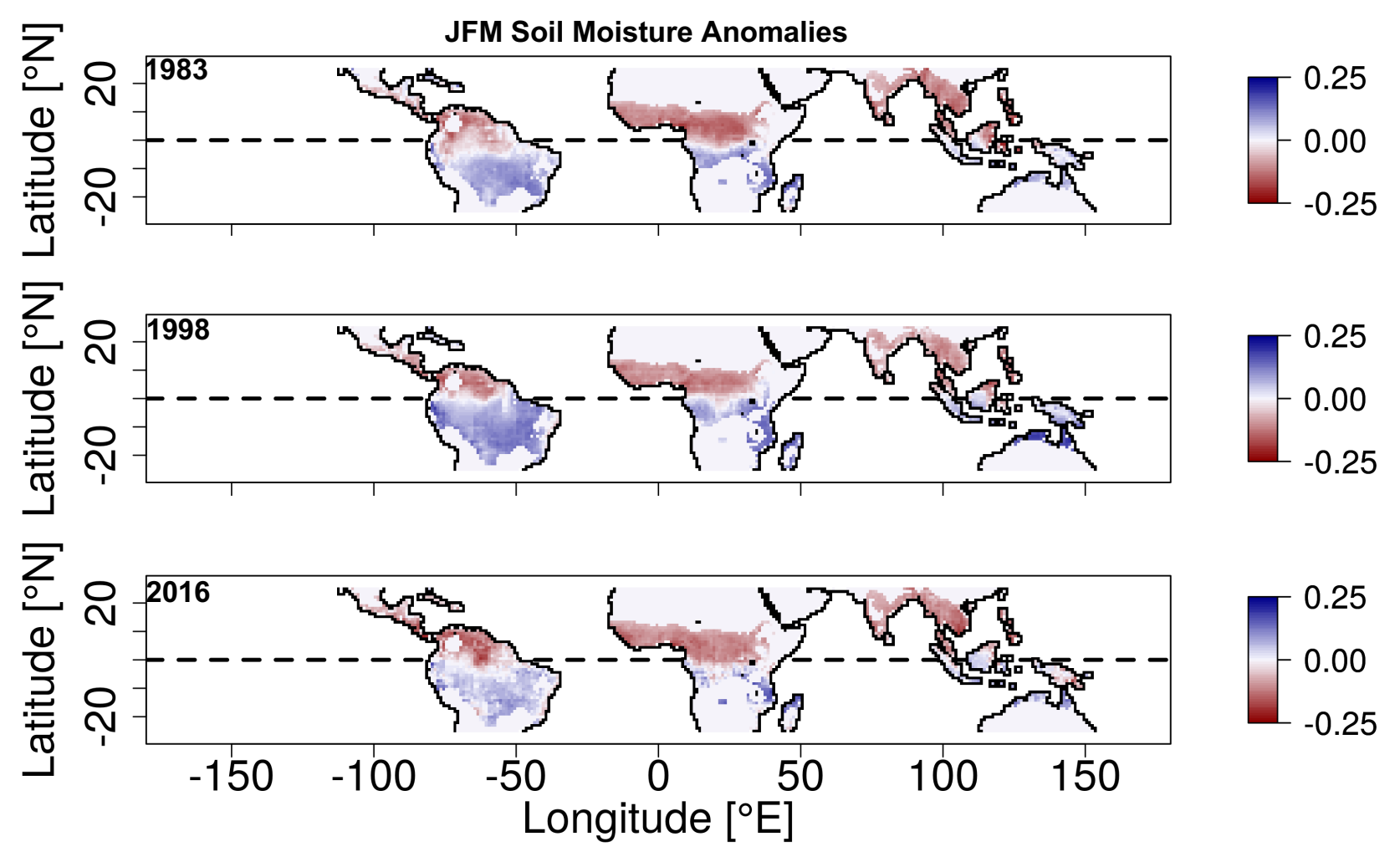

Figure S1b: Same as Figure S1a, but for January to March (JFM) in 1983 (top), 1998 (middle) and 2016 (bottom). 

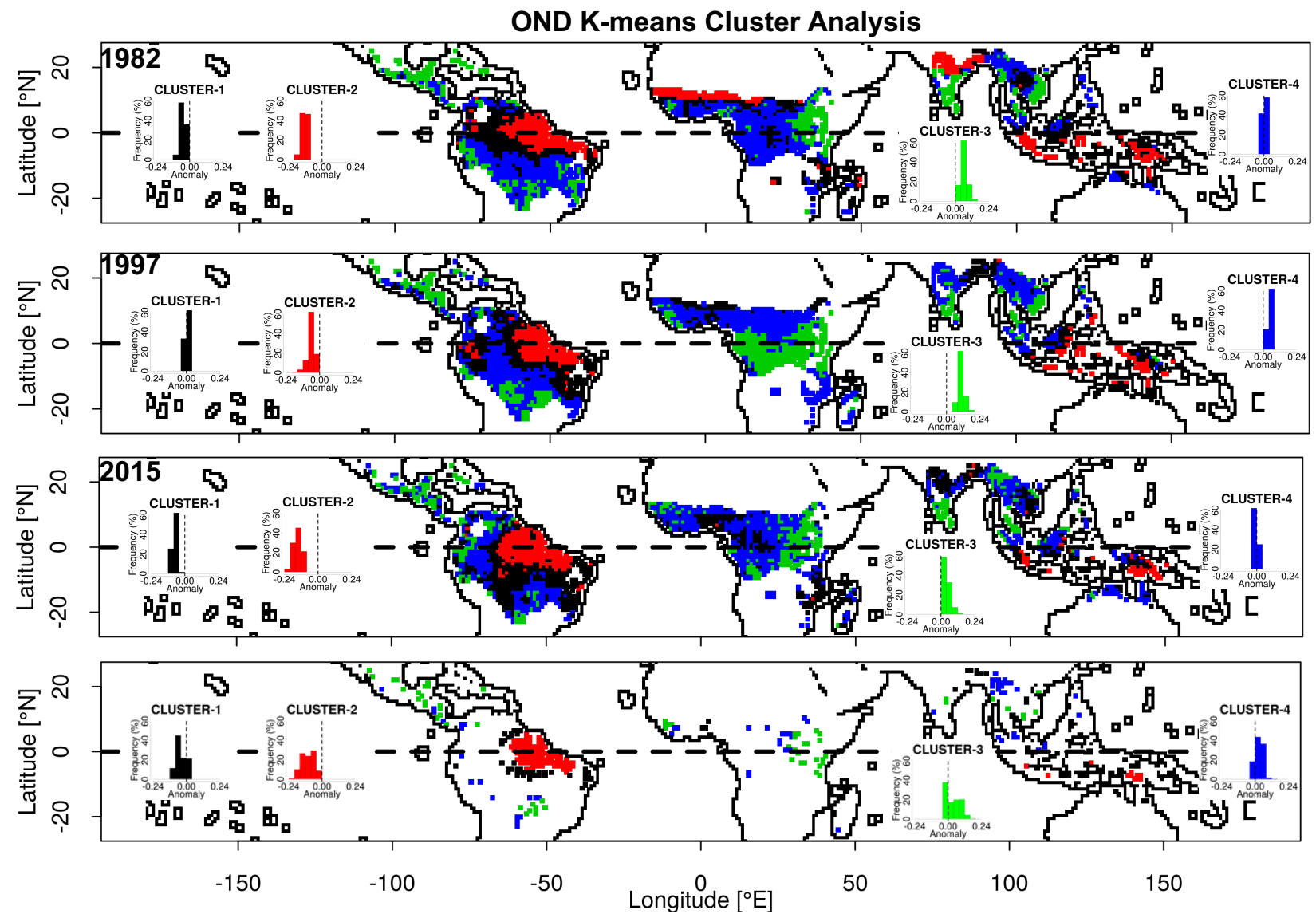

Figure S2a: K-means cluster analysis results October to December (OND) for 1982, 1997 and 2015 El Niños and the overlap of the three periods (top to bottom). Corresponding histograms of soil moisture anomalies for each of the four clusters also shown. Anomalies relative to 1979-2016 period. 

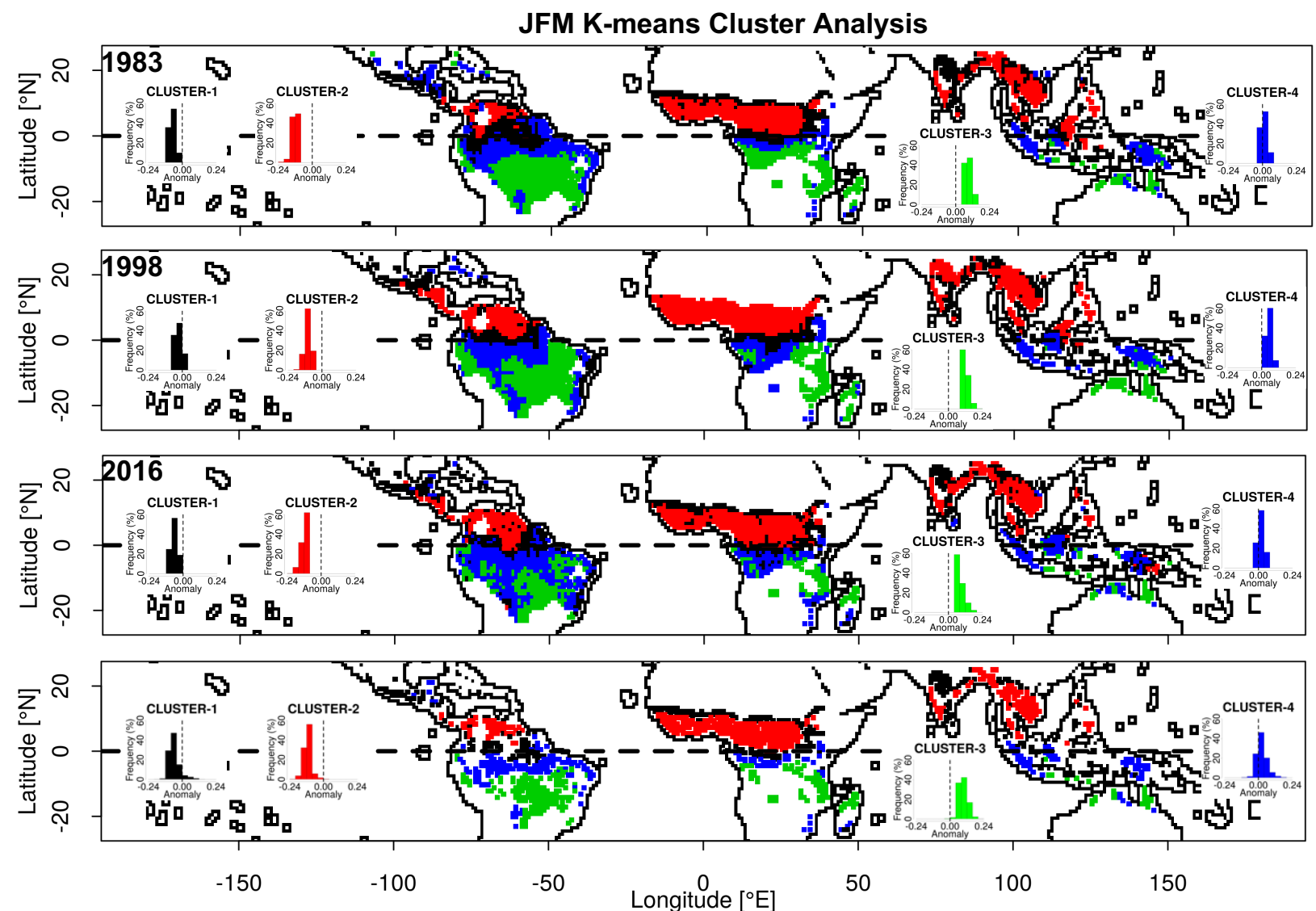

Figure S2b: Same as Figure S2a, but for January to March (JFM) for 1982, 1997 and 2015 and the overlap of the 3years (top to bottom). 


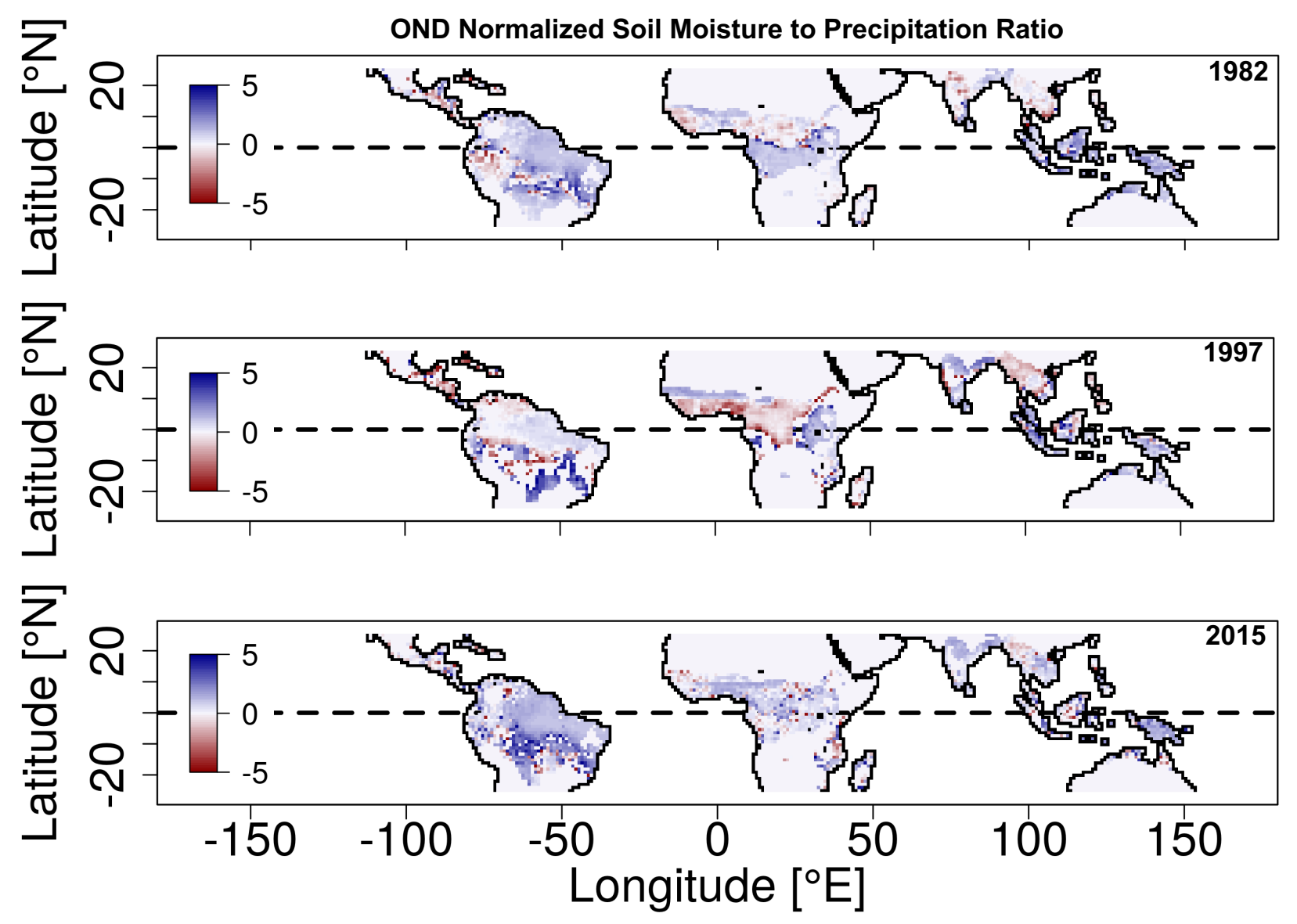

Figure S3a: Ratio of GLDAS soil moisture to precipitation change computed using October to December (OND) anomalies during El Niño years 1982-83, 1997-98, and 2015-16 relative to previous years. Anomalies normalized by the mean relative to $1979-2016$ period. 

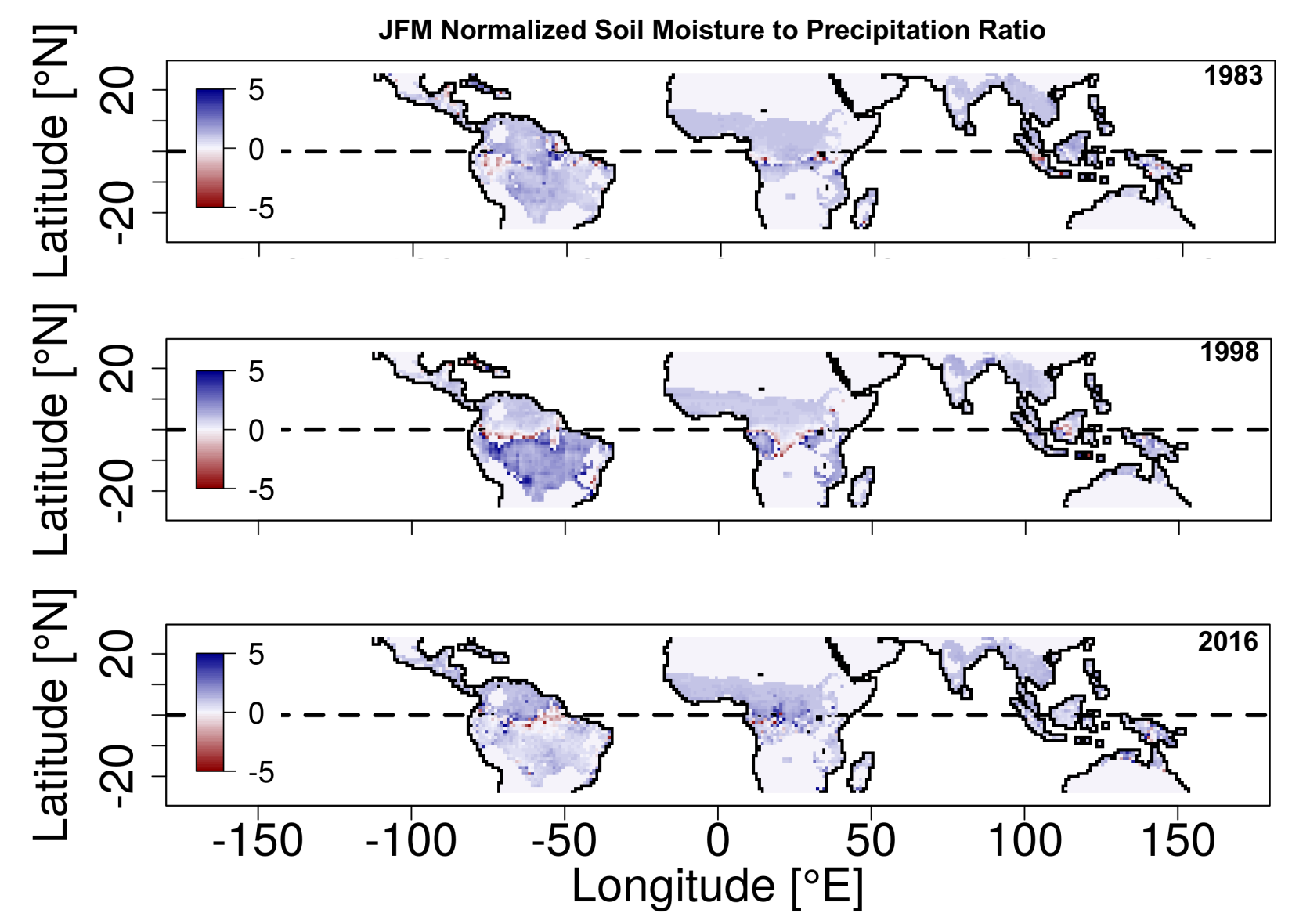

Figure S3b: Same as Figure S3a but for January to March in 1983 (top), 1998 (middle) and 2016 (bottom). 


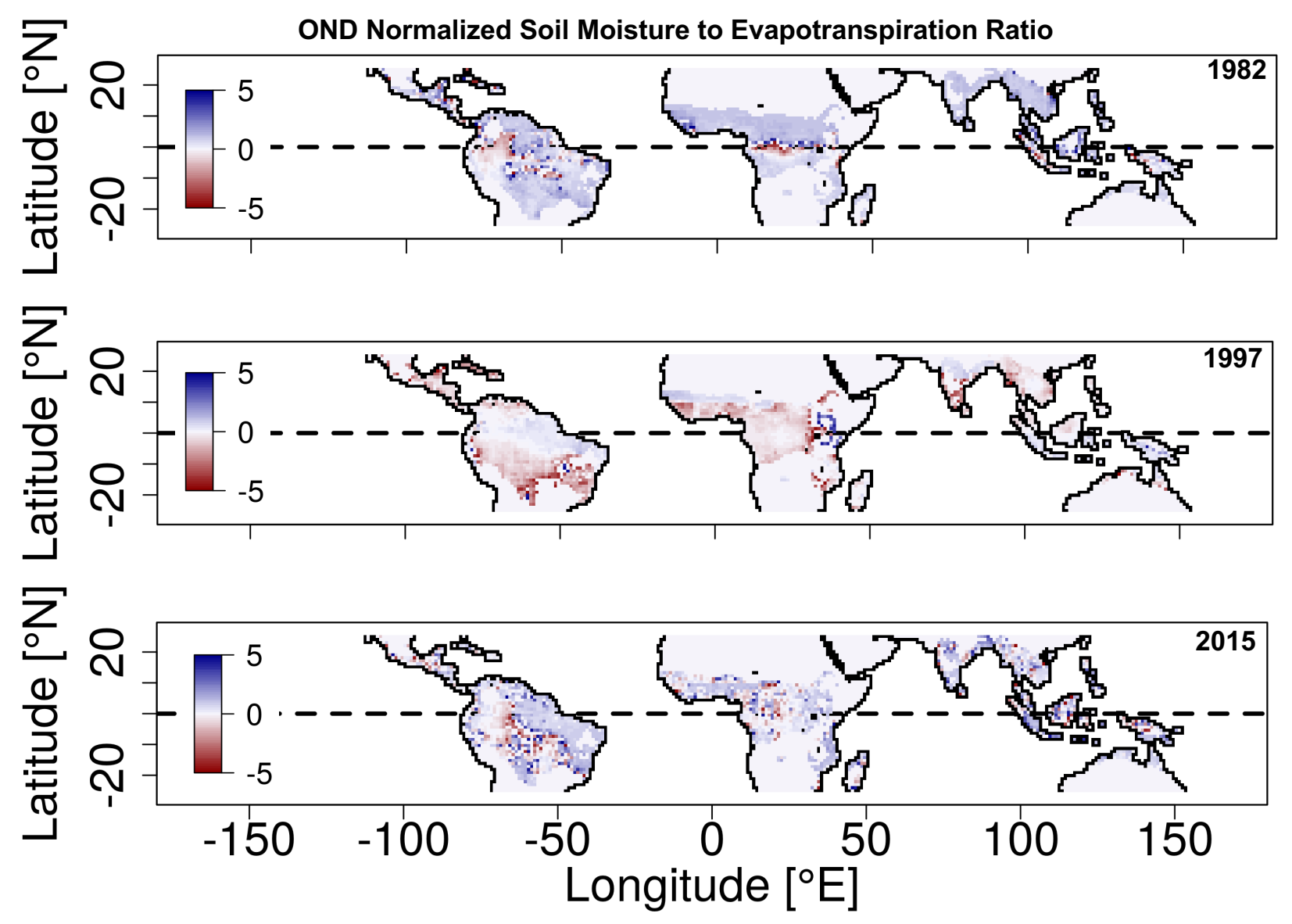

Figure S4a: Ratio of GLDAS soil moisture to evapotranspiration change computed using October to December (OND) anomalies during El Niño years 1982-83, 1997-98, and 2015-16 relative to previous years. Anomalies normalized by the mean relative to $1979-2016$ period. 


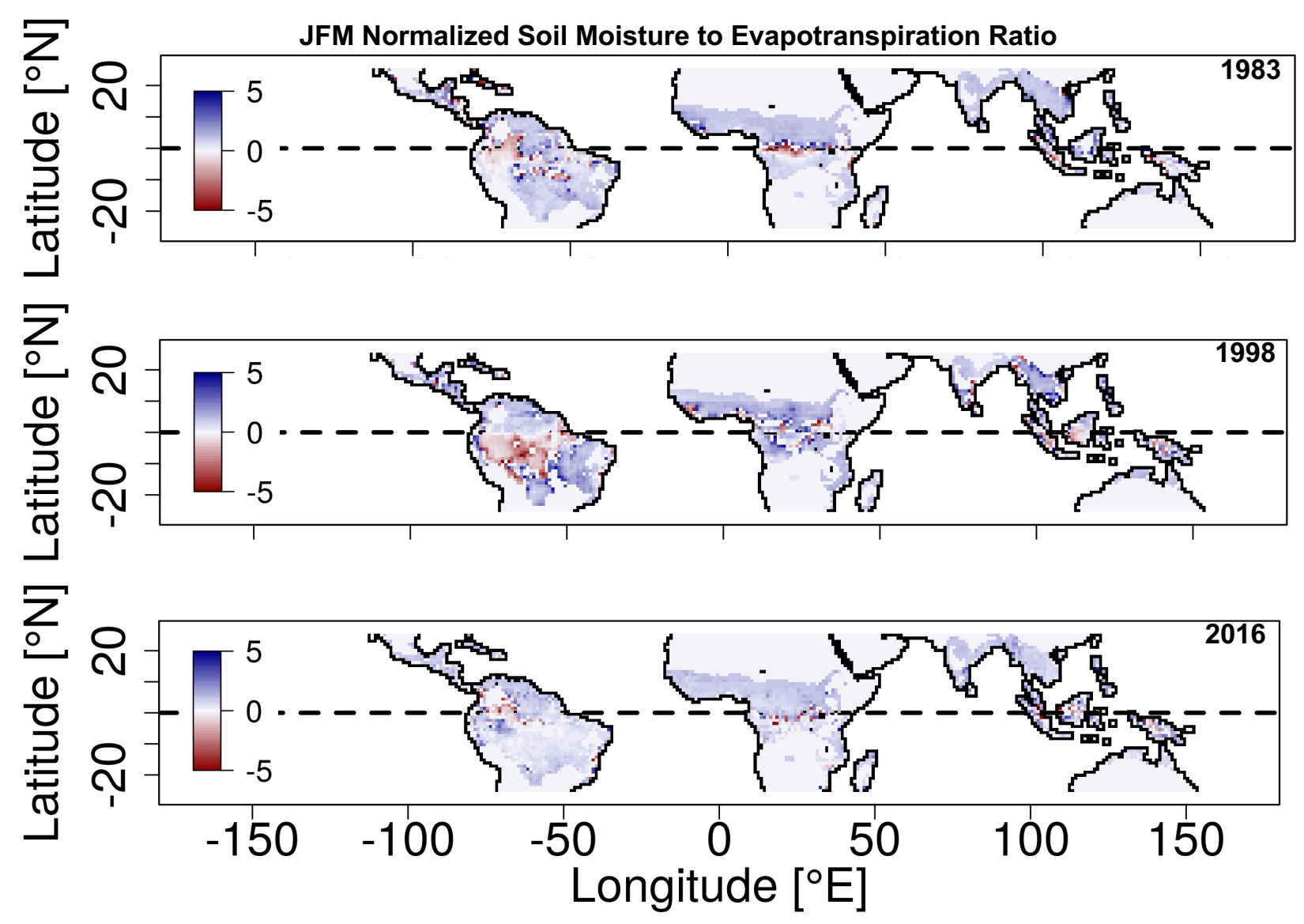

Figure S4b: Same as Figure S4a but for January to March in 1983 (top), 1998 (middle) and 2016 (bottom). Anomalies normalized by the mean relative to 1979-2016 period. 


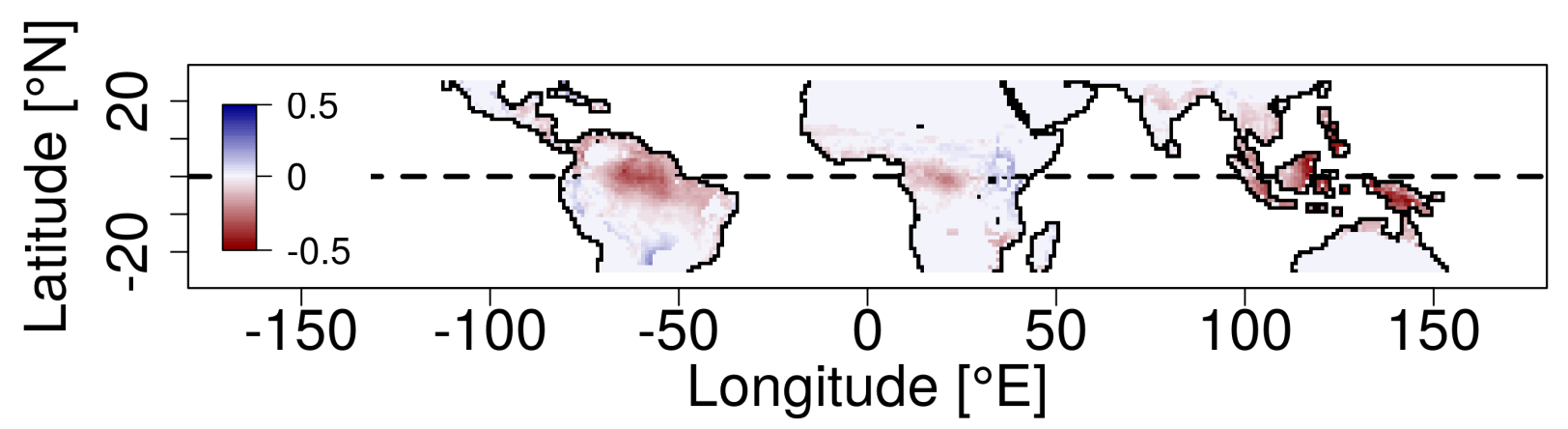

Figure S5: Pearson correlation coefficient between GLDAS soil moisture and NINO3.4 index from 1979 to 2016. Colors indicate regions where the mean correlation was negative (red) and positive (blue). 